\title{
A mode selection algorithm for mitigating interference in D2D enabled next- generation heterogeneous cellular networks
}

\author{
Md Kamruzzaman ${ }^{1}$, Nurul I Sarkar ${ }^{1}$, Jairo Gutierrez ${ }^{1}$, and Sayan Kumar Ray ${ }^{2}$ \\ ${ }^{1}$ Department of IT and Software Engineering, Auckland University of Technology, Auckland, New Zealand \\ ${ }^{2}$ Faculty of Business and Information Technology, Manukau Institute of Technology, Auckland, New Zealand. \\ \{zkamruzz,nurul.sarkar,jairo.gutierrez\}@aut.ac.nz,sayan.ray@mit.ac.nz
}

\begin{abstract}
Device-to-Device (D2D) communication is becoming more popular research topic among the network researchers and engineers across the globe This popularity is due to various key applications of D2D communications including 4G LTE-A envisioning the continuous evolution of current cellular networks into 5G to support a much larger and diverse range of applications. D2D communications play an important role to fulfill the quality of service (QoS) requirements for $5 \mathrm{G}$ networks. However, to realize the performance gain of D2D communications, various open challenges need to be resolved including mode selection (MS). This paper, therefore proposes a new mode selection algorithm for D2D enabled heterogeneous 5G cellular network. By quantifying the key parameters we mitigate interference that affect the system performance, and finally proposed an algorithm for optimal selection of transmission mode in a heterogeneous cellular network. The findings reported in this paper provide some insights into the design of $5 \mathrm{G}$ networks that may help network designer and engineers to contribute further towards the development of next generation wireless networks.
\end{abstract}

Keywords-Device to Device communication, D2D, Mode Selection, HetNets, interference management Public Safety.

\section{INTRODUCTION}

In cellular network, Device-to-Device (D2D) communication is an emerging technology where two proximity mobile UEs (User Equipment) communicate each other without any Base Station (BS) or core network. Due to the short communication range between a D2D pair, D2D communication provides several advantages in terms of spectrum efficiency, throughput, delay, power management, coverage expansion, and capacity improvement by reusing radio resources and allowing network functionalities to the devices.

Furthermore, D2D communication enables new services such as public safety, location-based commercial proximity, content sharing like files, videos or pictures, gaming, connectivity extension, and traffic offloading [1]. Owing to these benefits, D2D communication is being considered one of the key technologies in next-generation networks.

Last decade experienced an unexpected growth in both mobile broadband traffic as well as end-user demand for faster accessing the data. According to the latest Cisco visual networking index [2], mobile data traffic was grown up 18-fold, 400 petabytes per month in 2011 to 7.2 Exabyte per month at the end of 2016. It is expected to grow further sevenfold between 2016 and 2021, reaching 49.0 Exabyte per month by 2021.

Besides these, in 3rd Generation Partnership Project (3GPP) Release 12 for proximity-based services (ProSe) and group communication system enablers (GCSE) D2D communication is an integrated module in Forth Generation (4G) Long Term Evolution (LTE) Advanced standard. During natural disasters like earthquake or hurricane, with the help of D2D functionality, a replacement of the traditional network can be set up within a short period. Furthermore, multi-hop cooperation between devices can help to enhance the coverage which may be the only mode of communication in case of none coverage area as well as emergency. Hence, D2D communications play important role for traffic off-loading, improving spectrum efficiency and latency, capacity and coverage enhancements, and energy efficiency in the future cellular network [3].

However, to maximize the performance gain of D2D communication, there are many open challenges that need to be thoroughly addressed [1]. These challenges include mode selection, neighbor discovery, interference and radio resource management, energy consumption, the coexistence of D2D with small cells, mobility management, and network security. Among them, mode selection (MS) in a heterogeneous scenario comparing all tiers simultaneously is very important and complex.

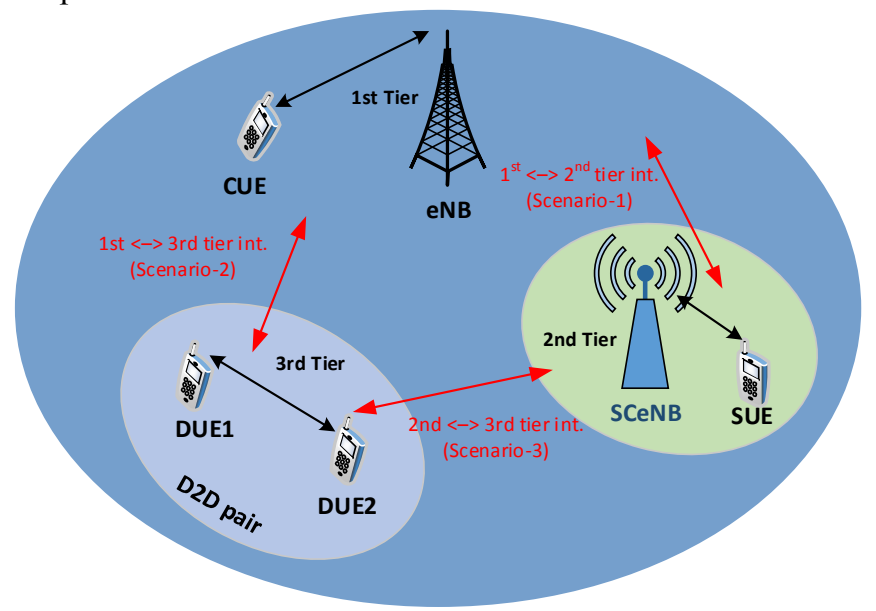

Figure 1: Interference in D2D communication in 3-tier networks

Figure 1 shows the possible interference scenario in a 3-tier cellular network like interference between the $1^{\text {st }}$-tier and the 
$2^{\text {nd }}$-tier, the $1^{\text {st }}$-tier and the $3^{\text {rd }}$-tier, and interference between the $2^{\text {nd }}$-tier and $3^{\text {rd }}$-tier are. Hence, to avail the benefits of D2D communication in cellular network interference management is essential and by selecting an appropriate mode of transmission we can achieve this. In spectrum sharing scenario there are two modes of operation either cellular mode (CM) where DUEs exchange the information via eNB by using orthogonal spectrum or sharing mode (SM) where CUE and DUE both share the same spectrum resources.

\section{RELATED WORK}

The state-of-the-art researchers proposed various methods for interference management in D2D enabled 2-tier cellular network such as split frequency, selection of transmission mode, power control, resource allocation, cognitive radio, game theoretic etc., and their working principles as well as techniques are also different.

Cross-tier and co-tier interference both can be avoided by using proper mode selection (MS) algorithm. In the-state-of-art work, many researchers focus on MS with different like path loss [4], based on received signal strength over the D2D link [5] or the physical distance among the devices [6-8]. However, these schemes do not reflect exact channel quality or interference issues.

In $[9,10]$ MS has been performed based on the channel quality. In particular [10], P. Janis et al. consider the sum rate of the connection between D2D pairs as well as CUEs as the MS criterion. In [11], Fan et al. worked on mode selection and resource allocation issues for D2D communications. In their proposal, they ensured the proper transmission of cellular users and derived under what conditions D2D underlay mode can be used by maintaining the channel quality good enough for CUEs. But the main constraint of this proposal is they considered all CUEs and D2D pairs are located in a single cell.

In [12], a distributed joint mode selection and power control scheme is proposed in an iterative manner by using Monte Carlo method. Based on effective interference on each RB (Resource Block), a D2D pair selects its mode and adjusts power to achieve the targeted throughput while CUEs performance will be unaffected. Like [11], their proposed scheme is only validated in a single cell but different radius.

By using the real-time information of local traffic channel and surrounding nodes, a distributed approach for a smart mode and power selection is proposed in[13]. Here dynamic switching is adopted between D2D transmissions, when the interference is limited otherwise continue the communication using cellular mode.

To resolve the issues arising from spectrum sharing in D2D enabled cellular network, a location related strategy for mode selection and spectrum sharing algorithm is proposed in[14] where devices form a coalition to share the spectrum among DUEs and CUEs.
All the aforementioned state-of-the-art techniques describe how to control interference in single or 2-tier cellular networks. Moreover, most of the MS or resource allocation schemes in the above literature consider only one D2D pair and both of them are in the same cell. Very few papers focus where DUEs in a D2D pair are attached to different eNBs. But in practice, multiple CUEs may associate with single eNB and form multiple D2D pairs. Not only multi-cell but in future a large number of small cells, especially femtocells will be deployed into the cellular network ( $5 \mathrm{G}$ and beyond) which will create a multi-tier network. In that multi-tier network the most prominent challenge is how to minimize the interference by selecting appropriate mode of transmission.

Despite the fact, there are very few on-going research efforts to address the MS as well as resource allocation for interference problems in 3-tiers cellular networks. Future D2D enabled cellular network will be heterogeneous and the complete distributed solutions for the mode selection as well as radio resource allocation problems in a D2D-enabled multi-tier scenario has not been studied comprehensively in the literature. Nonetheless, none of the authors examines the worst and, at the same time, the most challenging interference case, where all 3tiers of the networks (i.e. eNB, SCeNB and D2D communication) mutually interfere. The main difference between the exiting approach and our proposal is mode selection in a 3-tier heterogeneous network.

\section{Proposed Mode SELECTION AlgORITHM For INTERFERENCE MITIGATION}

For an effective D2D communication in a heterogeneous cellular network considering the impact of interference from all 3-tiers simultaneously, our techniques will work on two phases. Firstly, based on the proposed model, select the optimal mode of transmission for minimizing interference. In the analytical model, we will first develop a mode selection mechanism (Figure-2) and finally construct a resource allocation model to quantify the performance gain for uplink/downlink communication in D2D-enabled multi-tier cellular networks.

In a multi-tier network, co-tier, and cross-tier both interferences exist. Therefore, designing the optimal network geometry model is an important issue to overcome the interference problem in dense HetNets. For design and analysis of cellular networks deterministic and stochastic geometry approaches are used. Deterministic methods are suitable only for topologies with fixed cell sizes not for heterogeneous network topology. On the other hand, stochastic geometry is a promising approach to predict probabilistic parameters of randomly designed HetNets such as SINR (signal-to-interference-plus-noise ratio), transmission power, throughput, coverage probability and load distribution [15]. 


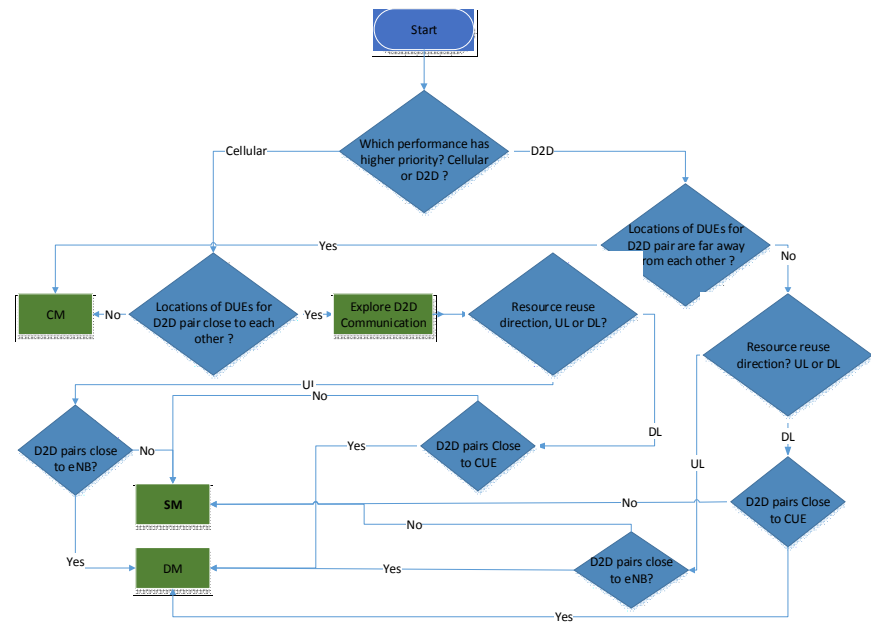

Figure 2: Flowchart of the proposed mode selection algorithm for D2D communications

In our proposal, we can use the stochastic geometry model to select the appropriate mode of transmission for D2d pairs. Different properties of a heterogeneous network like device location, the position of macro and small cells, the mobility of user etc. are considered as a random (stochastic) process of specified probability distribution [16]. To determine the spatial distribution of BS and users, we will use the Poisson Point Process (PPP).

\section{A. Analytical Model}

For modeling a 3-tier cellular network let us consider a macro cell, eNB at the center of the coverage area which is surrounded by several small cells. Small cells and UEs are assumed to be randomly deployed in the cellular network. Further assumed that the channel between any two UEs is reciprocal, UEs are limited to half-duplex communication, and all UEs are synchronized with the macro eNB. Also, assume that all spatial coexistence transmission links between UEs are mutually independent and path-loss is dominated by multipath fading.

Therefore, for a random communication link i-j Signal-toInterference-Noise Ratio (SINR), $\gamma^{j}$ for a random receiver $\mathrm{j}$ can be expressed as

$\gamma_{j}=\frac{P_{i} G_{i j} d_{i j}^{-\alpha}}{I+N_{o}}-----(1)$

Where $\alpha$ is the path loss coefficient, I is the interference at the receiver $\mathrm{j}$, the distance between the transmitter, $\mathrm{i}$ to the receiver, $\mathrm{j}$ is $\mathrm{d}_{\mathrm{ij}}$, channel coefficient between these two random UEs is $\mathrm{G}_{\mathrm{ij} \text {. }}$

Here the complex noise at the receiver is modeled as Additive White Gaussian Noise (AWGN) with zero mean and variance $\mathrm{N}_{\mathrm{o}}$, the power of thermal noise density.

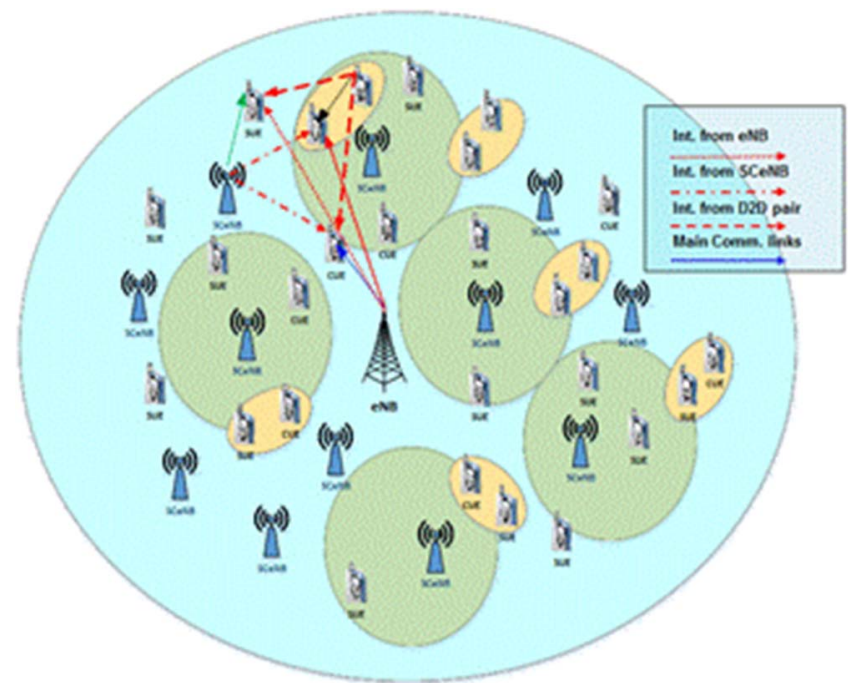

Figure 3: Interferences in a multi-tier network scenario

Therefore, the reliability of link i-j, $\mathrm{P}_{\text {rel }}$ can be written as $P_{r e l}=P_{r}\left(\gamma_{j} \geq \gamma_{t h}\right)------(2)$

Where $\gamma_{\text {th }}$ is the minimum required SINR for an effective communication.

Moreover, based on the Shannon theory, we can respectively derive the achievable data rates of that particular link, expressed as

$r=\log _{2}(1+\gamma)------(3)$

In an underlaying heterogeneous cellular network, for an effective D2D communication we have to fulfill the QoS for D2D link, a cellular link, and link under the small cell services. In D2D communication, D2D link shares cellular network resources and small cells also reuse the same resources. So in the service coverage area of macro cell $\mathrm{M}$ for any random D2D communication, for example, D2D link $\mathrm{i}-\mathrm{j}$, received signal will be interfered by the transmitting signals from macro eNB, M and small cell SCeNB, S in a Downlink (DL) sharing model.

Hence, the SINR at the receiver $\mathrm{j}$ of the $\mathrm{i}-\mathrm{j}$ D2D link can be expressed are

$\gamma_{j}^{D}=\frac{P_{D} G_{i j} d_{i j}^{-\alpha}}{I_{M}+I_{S}+N_{o}}-----(4)$

Where $\mathrm{P}_{\mathrm{D}}$ is the emission power of $\mathrm{D} 2 \mathrm{D}$ link transmitter, $\mathrm{I}_{\mathrm{M}}$ and $I_{S}$ are the interference due to the power emission of macrocell and small cell. $I_{M}=P_{M} G_{M j} d_{M j}^{-\alpha}$ and $I_{S}=\sum_{I \in S} P_{S} G_{i j} d_{i j}^{-\alpha}$ respectively. The transmitted power from eNB and SCeNB are $\mathrm{P}_{\mathrm{M}}$ and $\mathrm{P}_{\mathrm{S}}$ respectively.

After substituting these values into the above equation, the SINR $\gamma_{j}^{D}$ at the receiver of the D2D link can be represented as $\gamma_{j}^{D}=\frac{P_{D} G_{i j} d_{i j}^{-\alpha}}{P_{M} G_{M j} d_{M j}^{-\alpha}+\sum_{l \in S} P_{S} G_{l j} d_{l j}^{-\alpha}+N_{o}}----(5)$ 
TABLE 1. PARAMETERS USED IN THE SIMULATION

Thus, the reliability of the D2D link can be written as $P_{r e l}^{D}=P_{r}\left(\gamma_{j}^{D} \geq \gamma_{t h}\right)------(6)$

Similarly, for a random D2D links M-j in macro cell, D2D pairs receiver signal-to-interference-noise ratio, $\gamma_{j}^{M}$ can be expressed as

$\gamma_{j}^{D}=\frac{P_{M} G_{M j} d_{M j}^{-\alpha}}{P_{D} G_{D j} d_{D j}^{M-\alpha}+\sum_{l \in S} P_{S} G_{l j} d_{l j}^{-\alpha}+N_{o}}---(7)$

Where D2D link transmitter interference for the underlaying macro link, M-j is $I_{D}=P_{D} G_{D j} d_{D j}^{M-\alpha}$

And the reliability of the macro link $\mathrm{M} \rightarrow \mathrm{j}$ can be written as $P_{r e l}^{M}=P_{r}\left(\gamma_{j}^{M} \geq \gamma_{t h}\right)-------(8)$

In the heterogeneous cellular network, the link performance for all 3-tiers needs to be guaranteed. Let us consider a random UE $\mathrm{j}$ is associated with the small cell $\mathrm{S}_{0}$. In that case, the link from the small cell eNB to this UE S0 $\rightarrow \mathrm{j}$ will be interfered by the signals from other SCeNBs, $S^{\prime}=S / S_{0}$; eNB M of the macro cell where $\mathrm{S}_{0}$ belonging to and DUE transmitter of the D2D pair which is sharing its resources. Therefore, for the small cell, the SINR at the cellular $\mathrm{UE}_{\mathrm{j}}$ will be as

$$
\begin{aligned}
& \gamma_{j}^{S}=\frac{P_{S} G_{S_{0} j} d_{S_{0} j}^{-\alpha}}{I_{M}+I_{S}^{\prime}+I_{D}+N_{o}} \\
& =\frac{P_{S} G_{S_{0} j} d_{S_{0} j}^{-\alpha}}{P_{M} G_{M j} d_{M j}^{-\alpha}+\sum_{\substack{l \in S \\
l \neq S_{0}}} P_{S} G_{l j} d_{l j}^{-\alpha}+P_{D} G_{D j} d_{D j}^{S-\alpha}+N_{o}}--(9)
\end{aligned}
$$

Hence the reliability of small cell link $\mathrm{S}_{0} \rightarrow \mathrm{j}$ can be expressed as

$$
P_{r e l}^{S}=P_{r}\left(\gamma_{j}^{S} \geq \gamma_{t h}\right)------------10
$$

By analyzing equations (4) to (10) and solving them under PPP distribution in stochastic geometry, we can explore at various conditions which MS is causing minimum interference to the existing CUEs. We can also derive the distance requirement criteria for the D2D communication with optimal data rates by guaranteeing the cellular communication.

Once appropriate communication mode is selected for D2D communication, co-channel interference will be introduced due to resource reuse. One can further investigate the interference strategy by allocating cellular resource efficiently and guaranteeing QoS for cellular links and to maximize the system throughput.

\section{RESULTS AND DISCUSSION}

To get numerical results, we used a MATLAB tool with the initial parameters as shown in Table 1 .

\begin{tabular}{|l|l|l|}
\hline \multicolumn{1}{|c|}{ Symbol } & \multicolumn{1}{c|}{ Description } & \multicolumn{1}{c|}{ Values } \\
\hline$\alpha$ & Path loss exponent & 4 \\
\hline No & Power density spectrum & $-174 \mathrm{dBm}$ \\
\hline $\mathrm{P}_{\mathrm{M}}$ & Transmit power of eNB & $46 \mathrm{dBm}$ \\
\hline $\mathrm{P}_{\mathrm{S}}$ & Transmit power of SCeNB & $33 \mathrm{dBm}$ \\
\hline $\mathrm{P}_{\mathrm{D}}$ & Transmit power of DUEs & $13 \mathrm{dBm}$ \\
\hline
\end{tabular}

Figure 4 represents the CDFs (Cumulative Distribution Functions) of SINR for different links. We observe that by increasing the required SINR decreases the outage probability of D2D link sharply after a certain level whereas macro and small cell links can sustain further which represent the vulnerability of D2D link compare to cellular links with higher SINR requirement in a heterogeneous cellular network. Figure 5 shows that D2D link reliability increases while the distance between D2D pairs and eNB (macro and Small cell base station) increases and reciprocal while the distance between D2D pairs increases.

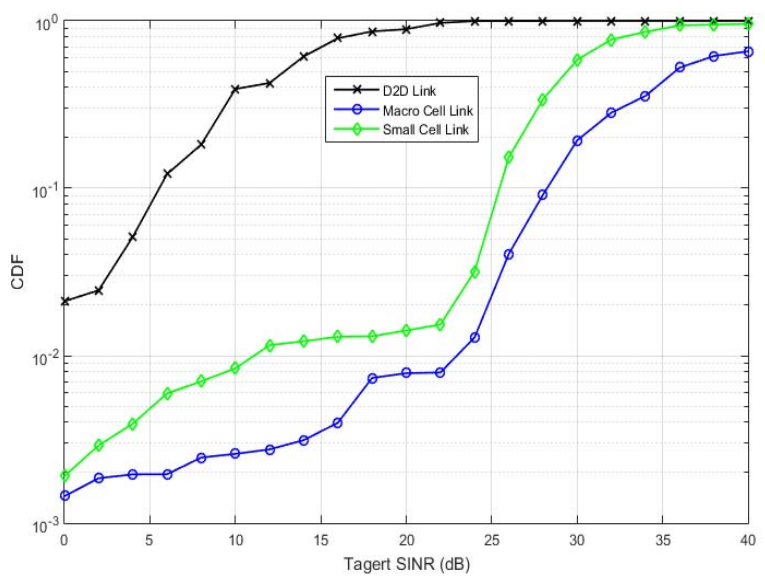

Figure 4: SINR for different links in the D2D communications



Figure 5: Impact of distance for various nodes in D2D communications

\section{CONCLUSIONS}

In this paper, we proposed a new mode selection algorithm for D2D enabled heterogeneous 5G cellular networks. These results are basic outcomes of our analytical model that linking the theoretical analysis of D2D communication in 3-tier cellular 
network. Results obtained have shown that the outage probability of D2D links sharply increased while participating DUEs are moving farther from each other whereas reciprocal while D2D pairs are moving away from the cellular base station. An investigation of D2D link reliability and throughput is suggested as future work.

\section{REFERENCES}

[1] X. Lin, J. G. Andrews, A. Ghosh, and R. Ratasuk, "An overview of 3GPP device-to-device proximity services," IEEE Communications Magazine, vol. 52, pp. 40-48, 2014.

[2] C. V. Networking Index, "Cisco Visual Networking Index: Global Mobile Data Traffic Forecast Update, 2016-2021 White Paper," ed,2017.

[3] H. A. U. Mustafa, M. A. Imran, M. Z. Shakir, A. Imran, and R. Tafazolli, "Separation Framework: An Enabler for Cooperative and D2D Communication for Future 5G Networks," IEEE Communications Surveys \& Tutorials, vol. 18, pp. 419-445, 2016.

[4] X. Hongnian and S. Hakola, "The investigation of power control schemes for a device-to-device communication integrated into OFDMA cellular system," in 21st Annual IEEE International Symposium on Personal, Indoor and Mobile Radio Communications, 2010, pp. 1775-1780.

[5] Z. Liu, T. Peng, S. Xiang, and W. Wang, "Mode selection for Device-to-Device (D2D) communication under LTEAdvanced networks," in 2012 IEEE International Conference on Communications (ICC), 2012, pp. 55635567.

[6] H. ElSawy, E. Hossain, and M. S. Alouini, "Analytical Modeling of Mode Selection and Power Control for Underlay D2D Communication in Cellular Networks," IEEE Transactions on Communications, vol. 62, pp. 41474161, 2014.

[7] X. Lin, J. G. Andrews, and A. Ghosh, "Spectrum Sharing for Device-to-Device Communication in Cellular Networks," IEEE Transactions on Wireless Communications, vol. 13, pp. 6727-6740, 2014.

[8] E. Frlan, "Direct communication wireless radio system," ed: Google Patents, 2000.

[9] C. H. Yu, O. Tirkkonen, K. Doppler, and C. Ribeiro, "On the Performance of Device-to-Device Underlay Communication with Simple Power Control," in VTC Spring 2009 - IEEE 69th Vehicular Technology Conference, 2009, pp. 1-5.

[10] P. Janis, Y. Chia-Hao, K. Doppler, C. Ribeiro, C. Wijting, H. Klaus, et al., "Device-to-device communication underlaying cellular communications systems," International Journal of Communications, Network and System Sciences, vol. 2, p. 169, 2009.

[11] F. Jiang, B. Wang, C. Sun, Y. Liu, and R. Wang, "Mode selection and resource allocation for device-to-device communications in 5G cellular networks," China Communications, vol. 13, pp. 32-47, 2016.

[12] E. Naghipour and M. Rasti, "A distributed joint power control and mode selection scheme for D2D communication underlaying LTE-A networks," in Wireless Communications and Networking Conference (WCNC), 2016 IEEE, 2016, pp. 1-6.

[13] F. Librino and G. Quer, "Distributed Mode and Power Selection for non-Orthogonal D2D Communications: a Stochastic Approach," IEEE Transactions on Cognitive Communications and Networking, 2018.

[14] H. Chen, X. Deng, M. Gao, L. Yang, L. Guo, and M. Chi, "Location Related Communication Mode Selection and Spectrum Sharing for D2D Communications in Cellular Networks," in 2018 International Conference on Intelligent Transportation, Big Data \& Smart City (ICITBS), 2018, pp. 169-173.

[15] M. Haenggi, Stochastic Geometry for Wireless Networks: Cambridge, UK: Cambridge University Press, 2012.

[16] H. S. Dhillon, R. K. Ganti, F. Baccelli, and J. G. Andrews, "Modeling and Analysis of K-Tier Downlink Heterogeneous Cellular Networks," IEEE Journal on Selected Areas in Communications, vol. 30, pp. 550-560, 2012. 